\title{
Stereotactic body radiation therapy for prostate cancer: what is the appropriate patient-reported outcome for clinical trial design?
}

\section{Jennifer Ai-Lian Woo ${ }^{1 \dagger}$, Leonard N. Chen ${ }^{1+}$, Hongkun Wang ${ }^{2}$, Robyn A. Cyr ${ }^{1}$, Onita Bhattasali ${ }^{1}$, Joy S. Kim ${ }^{1}$, Rudy Moures ${ }^{1}$, Thomas M. Yung ${ }^{1}$, Siyuan Lei ${ }^{1}$, Brian Timothy Collins s ${ }^{1}$, Simeng Suy ${ }^{1}$, Anatoly Dritschilo ${ }^{1}$, John H. Lynch ${ }^{3}$ and Sean P. Collins ${ }^{1}$ *}

${ }^{1}$ Department of Radiation Medicine, Georgetown University Hospital, Washington, DC, USA

2 Department of Biostatistics, Bioinformatics, and Biomathematics, Georgetown University, Washington, DC, USA

${ }^{3}$ Department of Urology, Georgetown University Hospital, Washington, DC, USA

\section{Edited by:}

Dwight E. Heron, University of Pittsburgh Cancer Institute, USA

Reviewed by:

Rachelle Lanciano, Delaware County Memorial Hospital, USA

Josephine Kang, Flushing Radiation

Oncology Services, USA

*Correspondence:

Sean P. Collins, Department of Radiation Medicine, Georgetown University Medical Center, 3800 Reservoir Road, N.W., Washington, DC 20007, USA

e-mail:spc9@georgetown.edu

${ }^{\dagger}$ Jennifer Ai-Lian Woo and Leonard N. Chen have contributed equally to this work
Purpose: Stereotactic body radiation therapy (SBRT) is increasingly utilized as primary treatment for clinically localized prostate cancer. Consensus regarding the appropriate patient-reported outcome (PRO) endpoints for clinical trials evaluating radiation modalities for early stage prostate cancer is lacking. To aid in clinical trial design, this study presents PROs over a 36-month period following SBRT for clinically localized prostate cancer.

Methods: Between February 2008 and September 2010, 174 hormone-naïve patients with clinically localized prostate cancer were treated with 35-36.25 Gy SBRT (CyberKnife, Accuray) delivered in 5 fractions. Patients completed the validated Expanded Prostate Cancer Index Composite (EPIC)-26 questionnaire at baseline and all follow-ups. The proportion of patients developing a clinically significant decline in each EPIC domain score was determined. The minimally important difference (MID) was defined as a change of one-half the standard deviation from the baseline. Per Radiation Therapy Oncology Group (RTOG) 0938, we also examined the patients who experienced a decline in EPIC urinary domain summary score of $>2$ points (unacceptable toxicity defined as $\geq 60 \%$ of all patients reporting this degree of decline) and EPIC bowel domain summary score of $>5$ points (unacceptable toxicity defined as $>55 \%$ of all patients reporting this degree of decline) from baseline to 1 year.

Results: A total of 174 patients at a median age of 69 years received SBRT with a minimum follow-up of 36 months. The proportion of patients reporting a clinically significant decline (MID for urinary/bowel are 5.5/4.4) in EPIC urinary/bowel domain scores was $34 \% / 30 \%$ at 6 months, $40 \% / 32.2 \%$ at 12 months, and $32.8 \% / 21.5 \%$ at 36 months. The patients reporting a decrease in the EPIC urinary domain summary score of $>2$ points was $43.2 \%(\mathrm{Cl}: 33.7 \%, 54.6 \%)$ at 6 months, $51.6 \%(\mathrm{Cl}: 43.4 \%, 59.7 \%)$ at 12 months, and $41.8 \%(\mathrm{Cl}: 33.3 \%, 50.6 \%)$ at 36 months. The patients reporting a decrease in the EPIC bowel domain summary score of $>5$ points was $29.6 \%(\mathrm{Cl}: 21.9 \%, 39.3 \%)$ at 6 months, $29 \%$ (Cl: $22 \%, 36.8 \%)$ at 12 months, and $22.4 \%(\mathrm{Cl}: 15.7 \%, 30.4 \%)$ at 36 months.

Conclusion: Following prostate SBRT, clinically significant urinary symptoms are more common than bowel symptoms. Our prostate SBRT treatment protocol meets the RTOG 0938 criteria for moving forward to a Phase III trial comparing it to conventionally fractionated radiation therapy. Notably, between 12 and 36 months, the proportion of patients reporting a significant decrease in both EPIC urinary and bowel domain scores declined, suggesting a late improvement in these symptom domains. Further investigation is needed to elucidate (1) which EPIC domains bear the greatest influence on post-treatment quality of life and (2) at what time point PRO endpoint(s) should be assessed.
Abbreviations: $\mathrm{ADT}$, androgen deprivation therapy; CT, computed tomography; CTCAE, common terminology criteria for adverse events; CTV, clinical target volume; DVH, dose-volume histogram; EBRT, external-beam radiation therapy; EPIC, expanded prostate index composite; GI, gastrointestinal; GTV, gross target volume;
GU, genitourinary; IMRT, intensity-modulated radiation therapy; MID, minimal important difference; PTV, planning target volume; QOL, quality of life; RTOG, radiation therapy oncology group; SBRT, stereotactic body radiation therapy; SD, standard deviation. 


\section{INTRODUCTION}

Stereotactic body radiation therapy (SBRT) is a new standard treatment option for clinically localized prostate cancer $(1,2)$. SBRT delivers high doses of radiation with precision to the prostate and adjacent tissues while minimizing radiation exposure to bladder and rectum $(3,4)$. Biochemical disease free survival has been shown to be high with $\operatorname{SBRT}(2,5)$, demonstrating toxicity comparable to conventionally fractionated radiation therapy despite greater doses per fraction and higher biologically effective doses (5-8). Presently, evidence supporting superior outcomes associated with any particular radiation treatment approach for localized prostate cancer remains limited (9). As a result, the choice of intervention is guided by the treatment's toxicity profile and the patient's subsequent quality of life (QOL) (10).

Due to the close proximity of the bladder and rectum to the prostate, urinary and bowel toxicities are unavoidable following prostate cancer radiotherapy. These toxicities are commonly employed as the co-primary endpoints for Phase II trials evaluating the suitability of a new treatment option for clinically localized prostate cancer $(11,12)$. The clinical significance of these toxicities is determined by their severity, duration, and associated bother. Toxicity grade is clinician-assessed utilizing the items from the National Cancer Institute's Common Terminology Criteria for Adverse Events (CTCAE). The incidence of late genitourinary (GU) and gastrointestinal (GI) toxicity ( $\geq$ grade 2) after external-beam radiation therapy ranges from 10 to $30 \%$, generally occurring within the first 3 years. Recent data suggest that many low grade toxicities may resolve with time, and analysis of actuarial incidence may over-estimate their clinical significance (13).

Physicians' assessment of treatment-associated toxicities is historically unreliable (14), and in fact may underestimate their severity $(15,16)$. Compared with physician-reported data, patient responses to validated questionnaires may better illustrate longitudinal trends in toxicity following radiotherapy (17). The Expanded Prostate Cancer Index Composite (EPIC)-26, a validated patientreported outcome (PRO) instrument that evaluates health-related QOL, has been utilized to compare prostate cancer treatments with similar efficacy but differing toxicity profiles $(10,18,19)$. Increasingly, PRO are integrated into clinical trial design (20), though interpretation of missing data $(21,22)$ and the selection of appropriate outcome measures complicate the meaningful use of PROs in the trial setting.

A key to utilizing PRO in clinical trials is determining thresholds for minimal important difference (MID) (23-25). An MID is the smallest difference in a questionnaire domain score, which patients perceive as a meaningful change (26). The MID for a given domain is important in determining the required number of patients for study recruitment and interpreting the questionnaire results. It varies depending on the specific domain questionnaire utilized and the demographics of the patient population being studied. The MID may be determined statistically or by comparison to results with a standard treatment (27). The most commonly used statistical approach is to utilize one-half SD of the baseline domain score (23), which is specific to the patient population being analyzed. Such a distribution approach has been criticized because it does not provide information on the clinical relevance of the observed change (26). In general, most approaches lead to MIDs that are $5-10 \%$ of the instrument range $(23-25,28)$.

What PRO endpoint should be utilized to determine if the toxicity profile of a new treatment is associated with a superior QOL? Radiation Therapy Oncology Group (RTOG) 0938 (http://www.rtog.org), a phase II trial comparing different SBRT hypofractionation regiments, compares urinary and bowel QOL 1 year following SBRT to that following conventionally fractionated external-beam radiation therapy (standard arm from RTOG 0415; 73.8 Gy in 41 fractions). In the opinion of the investigators, the percentage of patients with change in EPIC bowel domain score (baseline to 1-year) that was worse than five points and a change in EPIC urinary domain score (baseline to 1-year) that was worse than two points are felt to be clinically meaningful endpoints to assess for tolerability and safety. One year was chosen as a balance between a sufficient time to assess late toxicity with still adequate number of patients following up to minimize the impact of missing data.

To date, limited data are available on PROs following SBRT to aid in clinical trial design. The objective of this study is to report the urinary and bowel QOL outcomes following SBRT in patients with clinically localized prostate cancer. These PROs may in turn help inform selection of appropriate endpoints in the design of future clinical trials.

\section{MATERIALS AND METHODS PATIENT SELECTION}

Eligible patients had a diagnosis of prostate cancer and were treated per our institutional protocol. Risk category was defined using the D'Amico classification (9). Patients who received androgen deprivation therapy (ADT) were excluded from this study due to its known adverse effects on PROs (29). Institutional IRB approval was obtained for retrospective review of data that were prospectively collected in our institutional database.

\section{SBRT TREATMENT PLANNING AND DELIVERY}

Stereotactic body radiation therapy treatment planning and delivery were performed as previously described $(4,7)$. Gold fiducial markers were placed into the prostate using ultrasound guidance. Treatment plans were created using fused thin cut computed tomography (CT) images and high-resolution magnetic resonance (MR) images. The clinical target volume (CTV) included the prostate and proximal seminal vesicles. The planning target volume (PTV) included a $5 \mathrm{~mm}$ anterolateral expansion and a $3 \mathrm{~mm}$ posterior expansion around the CTV. A prescription dose of 35-36.25 Gy was delivered to the PTV in five fractions of 77.25 Gy over 1-2 weeks. The prescription isodose line was limited to $\geq 75 \%$. The bladder, membranous urethra, and rectum were contoured and evaluated with dose-volume histogram analysis during treatment planning. Target position was confirmed multiple times during each treatment with a minimum of three properly placed fiducials (4).

\section{FOLLOW-UP AND STATISTICAL ANALYSIS}

Patients completed the EPIC-26 (30) before treatment and during routine follow-up visits 1 month after the completion of SBRT, every 3 months for the first year, and then every 6 months for the 
second and third years. To minimize the impact of missing data, patients who missed follow-ups were contacted and asked to fill out the questionnaires. The EPIC-26 is a validated tool that measures urinary and bowel QOL (30). To statistically compare changes between two time points, the levels of responses were assigned a score and the significance of the mean changes in the scores was assessed by paired $t$-test. Responses to the EPIC-26 questionnaire were grouped by physiological domains and assigned numerical scores. The multi-item scale scores were transformed linearly to a 0-100 scale as recommended in the scoring instructions for the EPIC-26. Lower numbers corresponded to worsening function and increased bother. Wilcoxon Signed-Rank Test analysis was used to assess differences in QOL scores compared to baseline. Paired $t$-test was used to assess significance of the change in scores. The MID to assess for clinically significant change in HRQOL from baseline was set as half an SD (23). Per RTOG 0938, we also examined the percentage of patients who experienced a decline in EPIC urinary domain summary score of $>2$ points (unacceptable toxicity defined as $\geq 60 \%$ of all patients reporting this degree of decline) and EPIC bowel domain summary score of $>5$ points (unacceptable toxicity defined as $>55 \%$ of all patients reporting this degree of decline) from baseline to 1 year.

\section{RESULTS}

From February 2008 to September 2010, 174 hormone-naïve patients with clinically localized prostate adenocarcinoma were treated per our institutional SBRT monotherapy protocol. The patients were followed for a minimum of 36 months following SBRT (range: 37-69 months). The median patient age was 69 (4890) years (Table 1). $55.7 \%$ of patients self-identified as white and $39.1 \%$ as black. Forty-two percent of patients were D'Amico low risk, $52.9 \%$ of patients were intermediate risk, and $5.1 \%$ of patients were high risk. The median prostate volume was 37.3 (11.6-138.7) cc. Moderate to severe lower urinary tract symptoms (baseline AUA $\geq 8$, with a median baseline AUA of 7) were reported by $49.4 \%$ of patients prior to treatment (Table 2 ).

Ninety percent of patients were treated with $36.25 \mathrm{~Gy}$ in five $7.25 \mathrm{~Gy}$ fractions (Table 1). The median follow-up was 3.9 years. The median pre-treatment PSA of $6.0 \mathrm{ng} / \mathrm{ml}$ declined to a median 3 years post-treatment PSA of $0.3 \mathrm{ng} / \mathrm{ml}$. There were six biochemical failures, occurring in one low-risk patient, four intermediate-risk patients, and one high-risk patient. The overall 3-year actuarial biochemical relapse free survival was 95.9\%. No patients received ADT at any time during the first 3 years following SBRT.

Baseline EPIC summary scores are shown in Table 2 and mean changes in EPIC summary scores from baseline to 3 years of follow-up are shown in Table 3. The MID value for the urinary domain was 5.5 . The EPIC urinary summary score declined transiently at 1 month post-SBRT (mean change, -7.5) (Table 3; Figure 1A) and returned to near baseline by 3 months post-SBRT (mean change from baseline, -1.0) (Table 3; Figure 1A). This acute decline was both statistically $(p<0.0001)$ and clinically significant. A second late protracted decline occurred between 9 and 18 months (mean change from baseline at 12 months, -4.1) (Table 3; Figure 1A). The EPIC urinary summary score was close
Table 1 | Patient characteristics at baseline

\begin{tabular}{llc}
\hline & & $\begin{array}{c}\text { Patients } \\
\text { (N= 174) }\end{array}$ \\
\hline Age & Age $\leq 60$ & $13.80 \%$ \\
& $60<$ Age $\leq 70$ & $46.60 \%$ \\
Race & Age $>70$ & $39.70 \%$ \\
& White & $55.70 \%$ \\
Median pre-treatment PSA (ng/mL) & & $39.10 \%$ \\
Risk groups (D'Amico's) & Other & $5.20 \%$ \\
& Low risk & $6.0(1.8-32.5)$ \\
SBRT dose & Intermediate risk & $52.00 \%$ \\
& High risk & $5.20 \%$ \\
& 36.25 Gy & $90.20 \%$ \\
& 35 Gy & $9.80 \%$ \\
\hline
\end{tabular}

Table 2 | Pre-treatment quality of life (QOL) scores.

\begin{tabular}{lcccc}
\hline Baseline AUA score & \% Patients $(\boldsymbol{n}=\mathbf{1 7 4})$ & & \\
\hline $0-7$ (mild) & 50.6 & & \\
$8-19$ (moderate) & 44.8 & & \\
$>20$ (severe) & 4.6 & & \\
\hline Baseline EPIC-26 summary score & Mean & SD & MID \\
\hline Urinary domain & 89 & 11.06 & 5.5 \\
Bowel domain & 95 & 8.81 & 4.4
\end{tabular}

to baseline 3 years post-SBRT (mean change from baseline, -2.5 ) (Table 3; Figure 1A). The proportion of patients reporting a clinically significant decline in EPIC urinary domain scores was $34 \%$ at 6 months, $40 \%$ at 12 months, and $32.8 \%$ at 36 months (Table 4; Figure 2A). The patients reporting a decrease in the EPIC urinary domain summary score of $>2$ points was $43.2 \%$ (CI: $33.7 \%, 54.6 \%$ ) at 6 months, $51.6 \%$ (CI: $43.4 \%, 59.7 \%$ ) at 12 months, and $41.8 \%$ (CI: 33.3\%, 50.6\%) at 36 months (Table 5; Figure 2B).

The MID value for the bowel domain was 4.4. The EPIC bowel summary score declined transiently at 1 month (mean change, -9.4) (Table 3; Figure 1B) and experienced a second, more protracted decline between 9 and 18 months (mean change from baseline at 12 months, -2.9$)$. Bowel declines at 1 and 12 months were statistically significant $(p<0.0001)$; however, only the 1 month change met the threshold for clinically significant change. The EPIC bowel summary score were near baseline at 3 years postSBRT (mean change from baseline, -2.4) (Table 3; Figure 1B). The proportion of patients reporting a clinically significant decline in EPIC bowel domain scores was $30 \%$ at 6 months, $32.2 \%$ at 12 months, and $21.5 \%$ at 36 months (Table 4; Figure 3A). The patients reporting a decrease in the EPIC bowel domain summary score of $>5$ points was $29.6 \%$ (CI: $21.9 \%, 39.3 \%$ ) at 6 months, $29 \%$ (CI: $22 \%, 36.8 \%$ ) at 12 months, and $22.4 \%$ (CI: $15.7 \%, 30.4 \%$ ) at 36 months (Table 5; Figure 3B). 


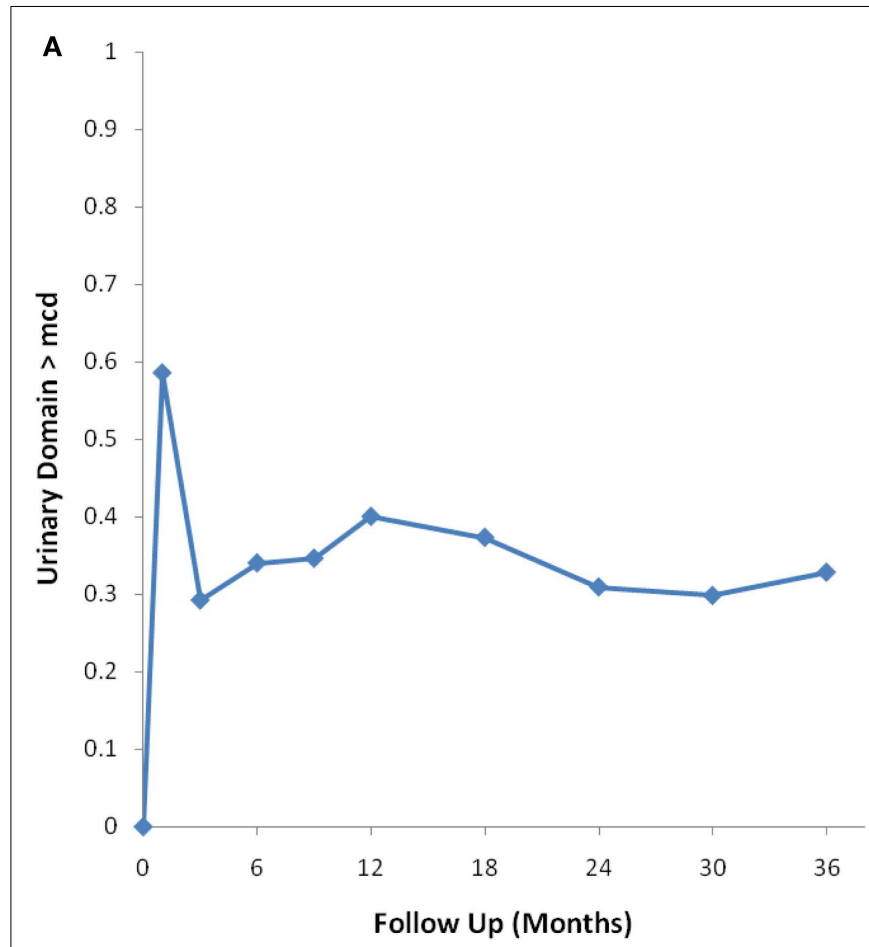

FIGURE 2 | Percentage of patients with a clinically significant decline in EPIC urinary summary domain scores following SBRT for prostate cancer. (A) Clinically significant decline defined as a one-half SD below

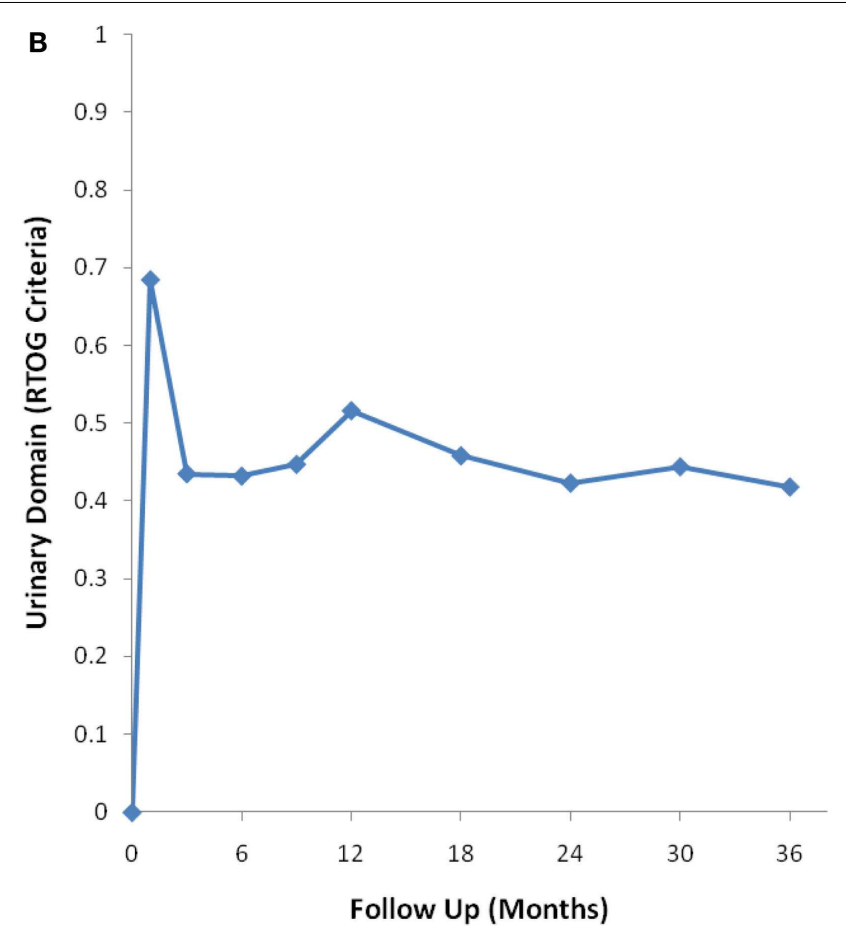

the baseline. (B) Clinically significant decline defined as a deviation in EPIC urinary domain summary score of $>2$ points below the baseline per RTOG 0938.

Table 5 | Proportion of patients with decrements that met RTOG 0938 criteria for significant declines in EPIC-26 domain scores following SBRT for prostate cancer.

\begin{tabular}{|c|c|c|c|c|c|c|c|c|c|c|}
\hline & Start & 1 month & 3 months & 6 months & 9 months & 12 months & 18 months & 24 months & 30 months & 36 months \\
\hline $\begin{array}{l}\text { Urinary domain } \\
\text { (decrease }>2 \text { pts } \\
\text { from baseline) }\end{array}$ & $\begin{array}{c}\text { Median }=91.7 \\
\text { Mean }=89.3\end{array}$ & $68.4 \%$ & $43.5 \%$ & $\begin{array}{c}43.2 \% \\
(33.7-54.6 \%)\end{array}$ & $44.7 \%$ & $\begin{array}{c}51.6 \% \\
(43.4-59.7 \%)\end{array}$ & $45.8 \%$ & $\begin{array}{c}42.3 \% \\
(34.3-50.7 \%)\end{array}$ & $44.4 \%$ & $\begin{array}{c}41.8 \% \\
(33.3-50.6 \%)\end{array}$ \\
\hline $\begin{array}{l}\text { Bowel domain } \\
\text { (decrease }>5 \text { pts } \\
\text { from baseline) }\end{array}$ & $\begin{array}{c}\text { Median }=100 \\
\text { Mean }=94.8\end{array}$ & $46.8 \%$ & $23.8 \%$ & $\begin{array}{c}29.6 \% \\
(21.9-39.3 \%)\end{array}$ & $30.2 \%$ & $\begin{array}{c}29.0 \% \\
(22.0-36.8 \%)\end{array}$ & $24.1 \%$ & $\begin{array}{c}24.8 \% \\
(18.1-32.5 \%)\end{array}$ & $29.2 \%$ & $\begin{array}{c}22.4 \% \\
(15.7-30.4 \%)\end{array}$ \\
\hline$N$ & 174 & 171 & 168 & 162 & 159 & 155 & 142 & 149 & 144 & 134 \\
\hline
\end{tabular}

In this study, we show that clinically significant urinary symptoms are more common than bowel symptoms over 36 months following prostate SBRT. Compared to RTOG 0415, the proportion of our patients with 1 year EPIC urinary domain declines $>2$ pts was higher (51.6 vs. $40 \%$ ). However, the proportion of our patients with 1 year EPIC bowel domain declines $>5$ pts was lower ( 29 vs. $35 \%$ ). Our patients were treated with 35 or 36.25 Gy in five fractions, which corresponds to a tumor equivalent dose in 2-Gy fractions (EQD2) of approximately 85-90 Gy assuming an $\alpha / \beta$ ratio of 1.5 . Considering this high BED and our inhomogeneous treatment plans (31), it is not surprising that the percentage of our patients experiencing an EPIC urinary domain score decline was higher than patients treated with low dose conventionally fractionated intensity-modulated radiation therapy (IMRT) (73.8 Gy) at 1 year after treatment. Unexpectedly, the percentage of our patients experiencing an EPIC bowel domain score decline was lower than patients treated on the control arm of RTOG 0415. We believe that this favorable bowel QOL profile with SBRT may be secondary to increased accuracy with intrafraction fiducial tracking and narrowed target volumes, thus sparing normal rectum from inadvertent irradiation. Which treatmentrelated symptom bears the greatest influence on post-treatment QOL? Utility analyses have shown that bowel symptoms have a greater negative impact on QOL than urinary symptoms or impotence (32). However, this may not apply for all patients, and shared decision making may be most appropriate (33).

An important finding of this study is that our prostate SBRT treatment protocol meets the RTOG 0938 criteria, suggesting that urinary and bowel QOL is not significantly worse following our SBRT approach compared with conventionally fractionated IMRT. 


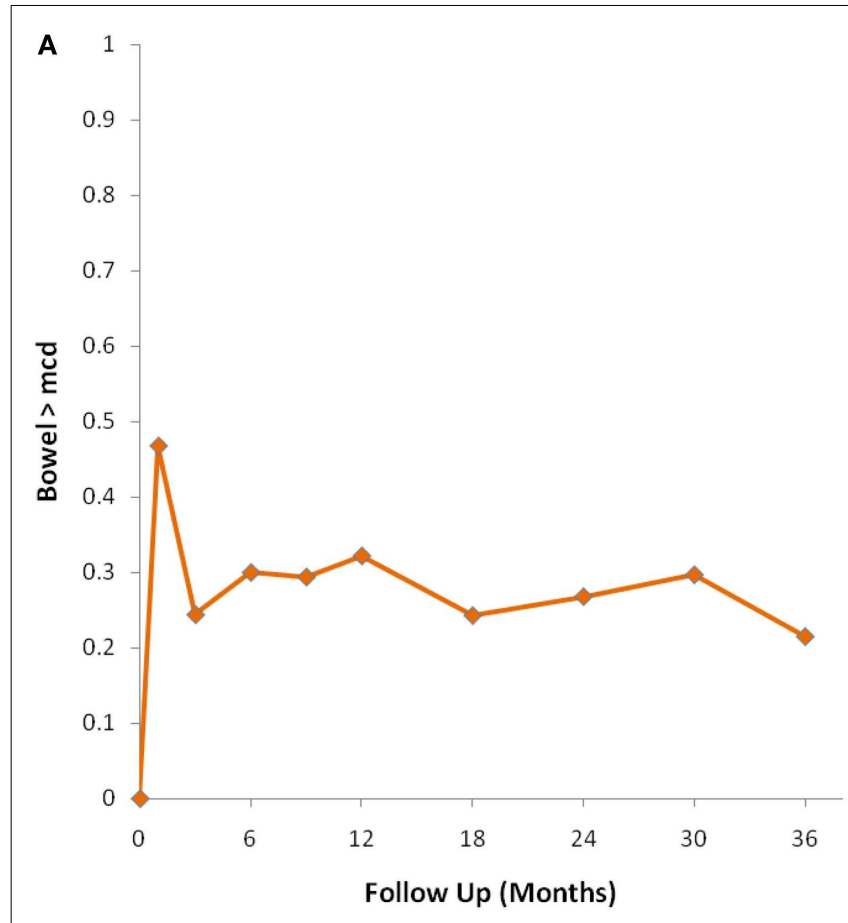

FIGURE 3 | Percentage of patients with a clinically significant decline in EPIC bowel summary domain scores following SBRT for prostate cancer. (A) Clinically significant decline defined as a one-half SD below

Based on patient preference for a shorter treatment course, SBRT utilization is likely to continue to increase as long as post-treatment QOL is comparable to conventionally fractionated IMRT.

At what time point should PRO endpoint(s) be assessed following prostate SBRT? Due to cost constraints, the timing of PRO assessments in Phase II trials are commonly limited to baseline and at one additional key time point that will determine whether to move the therapy forward to a Phase III trial. Acute toxicities usually resolve with time, but late toxicities commonly persist to cause a greater impact on long-term QOL. The length of follow-up (at least 36 months) in this cohort permitted us to capture a clinically meaningful difference in urinary and bowel symptoms, which may not be reflected by evaluating MID at the time point of 1 year per the existing RTOG protocol. Evaluation of PROs at a later time point beyond 1 year may yield more accurate assessment of long-term urinary and bowel QOL following radiation therapy. Recent evidence suggests that incorporation of web-based QOL survey technology in clinical trial design may further raise response rates, thus expanding opportunities to document even longer-term outcomes $(17,21)$.

\section{CONCLUSION}

Following prostate SBRT, clinically significant urinary symptoms are more common than bowel symptoms. Our prostate SBRT treatment protocol meets the RTOG 0938 criteria for moving forward to a Phase III trial comparison to conventionally fractionated radiation therapy. Notably, between 12 and 36 months, the proportion of patients reporting a significant decrease in both

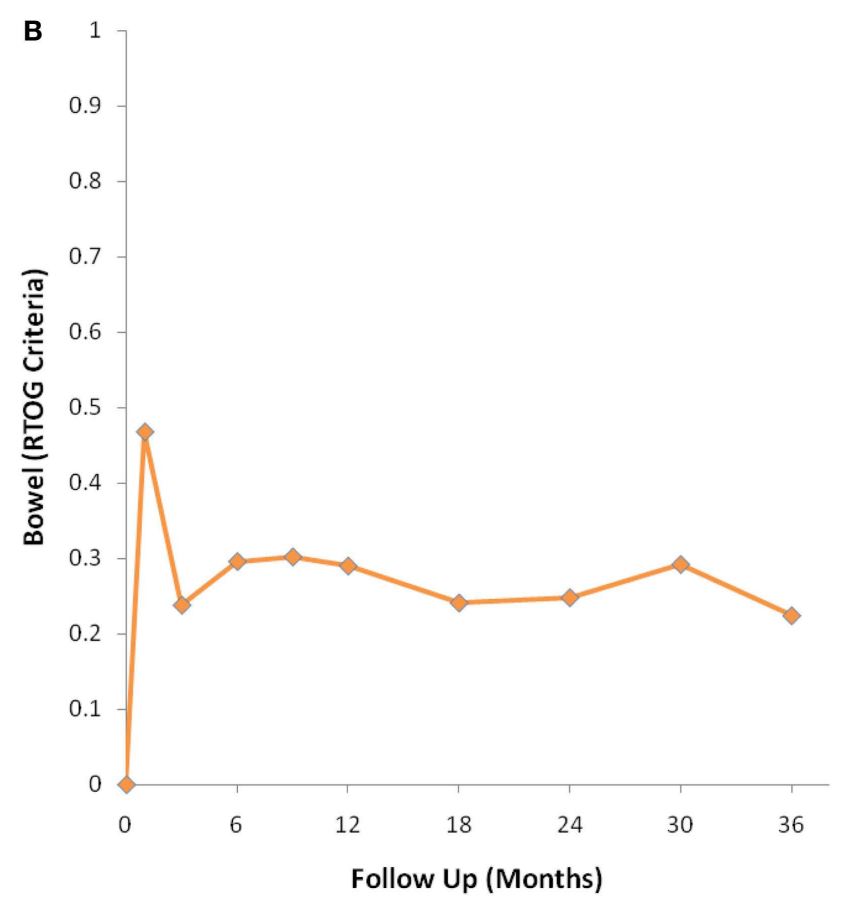

the baseline. (B) Clinically significant decline defined as a deviation in EPIC bowel domain summary score of $>5$ points below the baseline per RTOG 0938.

EPIC urinary and bowel domain scores declined, suggesting a late improvement in these symptoms.

\section{ACKNOWLEDGMENTS}

This work was supported by the James and Theodore Pedas Family Foundation.

\section{REFERENCES}

1. King CR, Collins S, Fuller D, Wang PC, Kupelian P, Steinberg M, et al. Healthrelated quality of life after stereotactic body radiation therapy for localized prostate cancer: results from a multi-institutional consortium of prospective trials. Int J Radiat Oncol Biol Phys (2013) 87:939-45. doi:10.1016/j.ijrobp.2013. 08.019

2. King CR, Freeman D, Kaplan I, Fuller D, Bolzicco G, Collins S, et al. Stereotactic body radiotherapy for localized prostate cancer: pooled analysis from a multiinstitutional consortium of prospective phase II trials. Radiother Oncol (2013) 109:217-21. doi:10.1016/j.radonc.2013.08.030

3. Xie Y, Djajaputra D, King CR, Hossain S, Ma L, Xing L. Intrafractional motion of the prostate during hypofractionated radiotherapy. Int J Radiat Oncol Biol Phys (2008) 72:236-46. doi:10.1016/j.ijrobp.2008.04.051

4. Lei S, Piel N, Oermann EK, Chen V, Ju AW, Dahal KN, et al. Six-dimensional correction of intra-fractional prostate motion with CyberKnife stereotactic body radiation therapy. Front Oncol (2011) 1:48. doi:10.3389/fonc.2011.00048

5. Katz AJ, Santoro M, Diblasio F, Ashley R. Stereotactic body radiotherapy for localized prostate cancer: disease control and quality of life at 6 years. Radiat Oncol (2013) 8:118. doi:10.1186/1748-717X-8-118

6. Freeman DE, King CR. Stereotactic body radiotherapy for low-risk prostate cancer: five-year outcomes. Radiat Oncol (2011) 6:3. doi:10.1186/1748-717X-6-3

7. Chen LN, Suy S, Uhm S, Oermann EK, Ju AW, Chen V, et al. Stereotactic body radiation therapy (SBRT) for clinically localized prostate cancer: the Georgetown University experience. Radiat Oncol (2013) 8:58. doi:10.1186/1748-717X-8-58

8. McBride SM, Wong DS, Dombrowski JJ, Harkins B, Tapella P, Hanscom HN, et al. Hypofractionated stereotactic body radiotherapy in low-risk prostate 
adenocarcinoma: preliminary results of a multi-institutional phase 1 feasibility trial. Cancer (2012) 118:3681-90. doi:10.1002/cncr.26699

9. D'Amico AV, Whittington R, Malkowicz SB, Schultz D, Blank K, Broderick GA, et al. Biochemical outcome after radical prostatectomy, external beam radiation therapy, or interstitial radiation therapy for clinically localized prostate cancer. JAMA (1998) 280:969-74. doi:10.1001/jama.280.11.969

10. Sanda MG, Dunn RL, Michalski J, Sandler HM, Northouse L, Hembroff L, et al. Quality of life and satisfaction with outcome among prostate-cancer survivors. N Engl J Med (2008) 358:1250-61. doi:10.1056/NEJMoa074311

11. Hsu IC, Bae K, Shinohara K, Pouliot J, Purdy J, Ibbott G, et al. Phase II trial of combined high-dose-rate brachytherapy and external beam radiotherapy for adenocarcinoma of the prostate: preliminary results of RTOG 0321. Int J Radiat Oncol Biol Phys (2010) 78:751-8. doi:10.1016/j.ijrobp.2009.08.048

12. Lawton CA, Yan Y, Lee WR, Gillin M, Firat S, Baikadi M, et al. Long-term results of an RTOG Phase II trial (00-19) of external-beam radiation therapy combined with permanent source brachytherapy for intermediate-risk clinically localized adenocarcinoma of the prostate. Int J Radiat Oncol Biol Phys (2012) 82:e795-801. doi:10.1016/j.ijrobp.2011.11.040

13. Peters LJ, Zagars GK. Neutron therapy in prostate cancer - is the therapeutic ratio improved? Int J Radiat Oncol Biol Phys (1995) 31:204-5. doi:10.1016/03603016(95)92200-J

14. Atkinson TM, Li Y, Coffey CW, Sit L, Shaw M, Lavene D, et al. Reliability of adverse symptom event reporting by clinicians. Qual Life Res (2012) 21:1159-64. doi:10.1007/s11136-011-0031-4

15. Basch E, Iasonos A, McDonough T, Barz A, Culkin A, Kris MG, et al. Patient versus clinician symptom reporting using the National Cancer Institute common terminology criteria for adverse events: results of a questionnaire-based study. Lancet Oncol (2006) 7:903-9. doi:10.1016/S1470-2045(06)70910-X

16. Sonn GA, Sadetsky N, Presti JC, Litwin MS. Differing perceptions of quality of life in patients with prostate cancer and their doctors. J Urol (2013) 189:S59-65. doi:10.1016/j.juro.2012.11.032

17. Basch E. New frontiers in patient-reported outcomes: adverse event reporting, comparative effectiveness, and quality assessment. Annu Rev Med (2014) 65:307-17. doi:10.1146/annurev-med-010713-141500

18. Hoppe BS, Michalski JM, Mendenhall NP, Morris CG, Henderson RH, Nichols $\mathrm{RC}$, et al. Comparative effectiveness study of patient-reported outcomes after proton therapy or intensity-modulated radiotherapy for prostate cancer. Cancer (2014) 120:1076-82. doi:10.1002/cncr.28536

19. Gray PJ, Paly JJ, Yeap BY, Sanda MG, Sandler HM, Michalski JM, et al. Patientreported outcomes after 3-dimensional conformal, intensity-modulated, or proton beam radiotherapy for localized prostate cancer. Cancer (2013) 119:1729-35. doi: $10.1002 / \mathrm{cncr} .27956$

20. Calvert M, Blazeby J, Altman DG, Revicki DA, Moher D, Brundage MD. Reporting of patient-reported outcomes in randomized trials: the CONSORT PRO extension. JAMA (2013) 309:814-22. doi:10.1001/jama.2013.879

21. Movsas B, Hunt D, Watkins-Bruner D, Lee WR, Tharpe H, Goldstein D, et al. Can electronic web-based technology improve quality of life data collection? Analysis of radiation therapy oncology group 0828. Pract Radiat Oncol (2014) 4:187-91. doi:10.1016/j.prro.2013.07.014

22. Siddiqui F, Liu AK, Watkins-Bruner D, Movsas B. Patient-reported outcomes and survivorship in radiation oncology: overcoming the cons. J Clin Oncol (2014) 32(26):2920-7. doi:10.1200/JCO.2014.55.0707

23. Norman GR, Sloan JA, Wyrwich KW. Interpretation of changes in health-related quality of life: the remarkable universality of half a standard deviation. Med Care (2003) 41:582-92. doi:10.1097/00005650-200305000-00007
24. Ringash J, O’Sullivan B, Bezjak A, Redelmeier DA. Interpreting clinically significant changes in patient-reported outcomes. Cancer (2007) 110:196-202. doi:10.1002/cncr.22799

25. Barrett B, Brown D, Mundt M, Brown R. Sufficiently important difference: expanding the framework of clinical significance. Med Decis Making (2005) 25:250-61. doi:10.1177/0272989X05276863

26. Jayadevappa R, Malkowicz SB, Wittink M, Wein AJ, Chhatre S. Comparison of distribution- and anchor-based approaches to infer changes in health-related quality of life of prostate cancer survivors. Health Serv Res (2012) 47:1902-25. doi:10.1111/j.1475-6773.2012.01395.x

27. Revicki D, Hays RD, Cella D, Sloan J. Recommended methods for determining responsiveness and minimally important differences for patient-reported outcomes. J Clin Epidemiol (2008) 61:102-9. doi:10.1016/j.jclinepi.2007.03.012

28. Skolarus TA, Dunn RL, Sanda MG, Chang P, Greenfield TK, Litwin MS, et al. Minimally important difference for the expanded prostate cancer index composite short form. Urology (2015) 85:101-6. doi:10.1016/j.urology.2014.08.044

29. Gay HA, Michalski JM, Hamstra DA, Wei JT, Dunn RL, Klein EA, et al. Neoadjuvant androgen deprivation therapy leads to immediate impairment of vitality/hormonal and sexual quality of life: results of a multicenter prospective study. Urology (2013) 82:1363-8. doi:10.1016/j.urology.2013.06.062

30. Wei JT, Dunn RL, Litwin MS, Sandler HM, Sanda MG. Development and validation of the expanded prostate cancer index composite (EPIC) for comprehensive assessment of health-related quality of life in men with prostate cancer. Urology (2000) 56:899-905. doi:10.1016/S0090-4295(00)00858-X

31. Woo JA, Chen LN, Bhagat A, Oermann EK, Kim JS, Moures R, et al. Clinical characteristics and management of late urinary symptom flare following stereotactic body radiation therapy for prostate cancer. Front Oncol (2014) 4:122. doi:10.3389/fonc. 2014.00122

32. Stewart ST, Lenert L, Bhatnagar V, Kaplan RM. Utilities for prostate cancer health states in men aged 60 and older. Med Care (2005) 43:347-55. doi:10.1097/01.mlr.0000156862.33341.45

33. Sommers BD, Beard CJ, D’Amico AV, Dahl D, Kaplan I, Richie JP, et al. Decision analysis using individual patient preferences to determine optimal treatment for localized prostate cancer. Cancer (2007) 110:2210-7. doi:10.1002/cncr.23028

Conflict of Interest Statement: Sean P. Collins and Brian Timothy Collins serve as clinical consultants to Accuray Inc. The Department of Radiation Medicine at Georgetown University Hospital receives a grant from Accuray to support a research coordinator. The other authors declare that they have no competing interests.

Received: 28 January 2015; accepted: 13 March 2015; published online: 31 March 2015. Citation: Woo JA-L, Chen LN, Wang H, Cyr RA, Bhattasali O, Kim JS, Moures R, Yung TM, Lei S, Collins BT, Suy S, Dritschilo A, Lynch JH and Collins SP (2015) Stereotactic body radiation therapy for prostate cancer: what is the appropriate patient-reported outcome for clinical trial design? Front. Oncol. 5:77. doi: 10.3389/fonc.2015.00077

This article was submitted to Radiation Oncology, a section of the journal Frontiers in Oncology.

Copyright (c) 2015 Woo, Chen, Wang, Cyr, Bhattasali, Kim, Moures, Yung, Lei, Collins, Suy, Dritschilo, Lynch and Collins. This is an open-access article distributed under the terms of the Creative Commons Attribution License (CC BY). The use, distribution or reproduction in other forums is permitted, provided the original author(s) or licensor are credited and that the original publication in this journal is cited, in accordance with accepted academic practice. No use, distribution or reproduction is permitted which does not comply with these terms. 